\title{
AVALIAÇÃO DO EFEITO DAS VARIÁVEIS DE PROCESSO NO BRANQUEAMENTO DE POLPA CELULÓSICA COM PERÓXIDO DE HIDROGÊNIO
}

\author{
EVALUATIONOFTHEEFFECTOF VARIABLESPROCESS OFCELLULOSIC PULPBLEACHING \\ WITH HYDROGEN PEROXIDE
}

\author{
Camila Juliane Marcondes ${ }^{1}$ Gustavo Ventorim² ${ }^{2}$ Rafael dos Santos ${ }^{3}$ Felipo Augusto Pereira ${ }^{4}$
}

\begin{abstract}
RESUMO
O mercado de polpa celulósica branqueada tem trabalhado de modo a promover níveis de alvura com valores de $92 \%$ ISO obedecendo, contudo, às normas ambientais e buscando processos menos agressivos. Assim, o dióxido de cloro adicional que seria necessário para a elevação da alvura tem sido substituído pelo peróxido de hidrogênio. No presente trabalho, objetivou-se avaliar a influência da variação da carga de peróxido de hidrogênio, pressão e temperatura na sequência de branqueamento $\mathrm{D}_{0}(\mathrm{E}+\mathrm{P}) \mathrm{D}_{1}(\mathrm{P}+\mathrm{O})$. Para isto foi utilizada polpa kraft industrial, proveniente de híbrido de Eucalyptus urophylla X Eucalyptus grandis, pré-deslignificada com oxigênio, cujas características inicias eram: alvura de $61,8 \%$ ISO, viscosidade de 36,9 cP e número kappa de 8,9 . O último estágio $(\mathrm{P}+\mathrm{O})$ foi realizado variando-se a carga de peróxido de hidrogênio $(3 \mathrm{~kg} / \mathrm{t}$ e $6 \mathrm{~kg} / \mathrm{t})$, a temperatura $\left(95^{\circ} \mathrm{C}\right.$ e $\left.105^{\circ} \mathrm{C}\right)$ e a pressão $(0 \mathrm{~atm}$ e $5 \mathrm{~atm})$. Para avaliar o efeito de tais variações nas propriedades da polpa celulósica realizaram-se os testes de viscosidade, alvura e reversão de alvura de acordo com normas TAPPI. De modo geral, o aumento da carga de peróxido de hidrogênio (de 3 para $6 \mathrm{~kg} / \mathrm{t}$ ) proporcionou um pequeno incremento nos valores de alvura e uma pequena redução da viscosidade da polpa celulósica. $\mathrm{O}$ aumento de $10^{\circ} \mathrm{C}$ na temperatura teve efeito positivo sobre o parâmetro de alvura, com acréscimo dos respectivos valores, por outro lado, para a viscosidade, os valores encontrados apresentaram tendência de redução. Para a variação de pressão de oxigênio observou-se também aumento da alvura e diminuição da viscosidade.
\end{abstract}

Palavras-chave: dióxido de cloro; peróxido pressurizado; número kappa.

\begin{abstract}
The bleached pulp market has been working to provide brightness levels at values of $92 \%$ ISO respecting, however, environmental standards and seeking for less aggressive processes. Then, the additional chlorine dioxide that would be necessary to lift brightness has been replaced by hydrogen peroxide. The present study aimed to evaluate the influence of hydrogen peroxide charge, pressure and temperature variation in the bleaching sequence $\mathrm{D}_{0}(\mathrm{E}+\mathrm{P}) \mathrm{D}_{1}(\mathrm{P}+\mathrm{O})$. It was used industrial kraft pulp, from hybrid of Eucalyptus urophylla X Eucalyptus grandis, oxygen-delignified, whose initial characteristics were: brightness $61.8 \%$ ISO, viscosity $36.9 \mathrm{cP}$ and kappa number 8.9 . The last stage $(\mathrm{P}+\mathrm{O})$ was performed varying the hydrogen peroxide charge $(3 \mathrm{~kg} / \mathrm{t}$ and $6 \mathrm{~kg} / \mathrm{t})$, temperature $\left(95^{\circ} \mathrm{C}\right.$ and $\left.105^{\circ} \mathrm{C}\right)$ and pressure $(0 \mathrm{~atm}$ and $5 \mathrm{~atm})$. To evaluate the effect of such variations over cellulosic pulp, viscosity, brightness and brightness reversion tests were carried out according to TAPPI standards. In general, increase of hydrogen peroxide charge

1 Engenheira Industrial Madeireira, Mestranda em Engenharia Mecânica, Faculdade de Engenharia de Guaratinguetá, Universidade Estadual Paulista, Av. Ariberto Pereira da Cunha, 333, Portal das Colinas, CEP 12.516-410, Guaratinguetá (SP), Brasil.ca_juliane@hotmail.com

2 Químico, Dr., Professor do Campus de Itapeva, Universidade Estadual Paulista, Rua Geraldo Alckmin, 519, Vila Nossa Senhora de Fátima, CEP 18409-010, Itapeva (SP), Brasil. ventorim@itapeva.unesp.br

3 Engenheiro Industrial Madeireiro, Dr., Professor do Departamento de Mecânica, Instituto Federal de São Paulo, Av. João Olímpio de Oliveira, 1561, Vila Asem, CEP 18202-000, Itapetininga (SP), Brasil. rafasantos01@ifsp.edu.br 4 Engenheiro Industrial Madeireiro, Universidade Estadual Paulista, Campus de Itapeva, Wood Pack Indústria e Comércio Ltda., Rua Tino Vitto, 270 - Distrito Industrial, CEP 18760-000, Cerqueira César, (SP), Brasil. felipo_ pereira@hotmail.com.br
\end{abstract}

Recebido para publicação em 3/06/2017 e aceito em 31/07/2017

Ci. Fl., v. 28, n. 3, jul. - set., 2018 
(from $3 \mathrm{~kg} / \mathrm{t}$ to $6 \mathrm{~kg} / \mathrm{t}$ ) caused a small increasing on brightness values and a small viscosity decreasing on the cellulosic pulp. The increase of $10^{\circ} \mathrm{C}$ in temperature had a positive effect on brightness parameter, increasing the respective values, on the other hand, for the viscosity analysis the achieved values showed a reduction tendency. For the variation of oxygen pressure it was also observed brightness increasing and viscosity decreasing.

Keywords: chlorine dioxide; pressurized peroxide; kappa number.

\section{INTRODUÇÃO}

O processo de branqueamento celulósico é uma das etapas mais poluentes dentro do setor de celulose e papel devido à geração de organoclorados. As crescentes exigências ambientais têm levado as indústrias a uma busca por novas tecnologias e processos baseados em modificações dos métodos convencionais (NAVARRO, 2004). Assim, o peróxido de hidrogênio $\left(\mathrm{H}_{2} \mathrm{O}_{2}\right)$ tem se tornado essencial nos sistemas de branqueamento da polpa kraft, pois seu uso melhora a eficiência do branqueamento livre de cloro elementar (Elemental Chlorine Free - ECF). O processo de branqueamento ECF realizado no mundo nos dias de hoje, não requer necessariamente a implementação do estágio com peróxido de hidrogênio pressurizado. No entanto, a criação do processo de branqueamento ECF-Light (que utiliza pouco dióxido de cloro $\mathrm{ClO}_{2}$ ) exige um estágio de poder de alvejamento maior que o peróxido de hidrogênio realizado em pressão atmosférica (ROBLES; SOUZA; LEPORINI FILHO, 2006; VENSON, 2008).

No branqueamento de polpa celulósica, o peróxido de hidrogênio reage lentamente em baixas temperaturas, necessitando longo tempo de reação. Uma maneira de acelerar o processo é aumentar a temperatura do sistema, entretanto, até $95^{\circ} \mathrm{C}$, ainda requer um longo tempo de reação. Segundo Kvaerner Pulping Technologies (1996), pressurizar o sistema é uma alternativa para diminuir o tempo de reação, uma vez que assim podem-se atingir altas temperaturas, as quais devem ser ponderadas, pois, quando muito elevadas, a degradação dos carboidratos também irá aumentar. O efeito do gás pressurizante é puramente físico e a pressão de reação tem efeito positivo até 5 atm (PEREIRA, 1995).

Neste contexto, o presente estudo teve como objetivo avaliar o efeito da carga de peróxido de hidrogênio, da temperatura e da pressão de oxigênio no branqueamento e na qualidade da polpa branqueada.

\section{MATERIAL E MÉTODOS}

\section{Material}

Foi utilizada polpa kraft industrial, proveniente de híbrido de Eucalyptus urophylla X Eucalyptus grandis, pré-deslignificada com oxigênio. As características inicias da polpa eram: alvura de $61,8 \%$ ISO, viscosidade de $36,9 \mathrm{cP}$ e número kappa de 8,9 .

\section{Branqueamento}

Utilizou-se a sequência de branqueamento ECF-Light $\mathrm{D}_{0}(\mathrm{E}+\mathrm{P}) \mathrm{D}_{1}(\mathrm{P}+\mathrm{O})$, em que $\mathrm{D}_{0}$ e $\mathrm{D}_{1},(\mathrm{E}+\mathrm{P})$ e $(\mathrm{P}+\mathrm{O})$ são siglas referentes aos estágios de dióxido de cloro, extração alcalina com peróxido de hidrogênio e peróxido de hidrogênio pressurizado, respectivamente. As condições gerais de branqueamento são apresentadas na Tabela 1. 
TABELA 1: Condições gerais da sequência de branqueamento.

TABLE 1: General conditions of the bleaching sequence.

\begin{tabular}{ccccc}
\hline \multirow{2}{*}{ Condições } & \multicolumn{5}{c}{ Estágios de Branqueamento } \\
\cline { 2 - 5 } & $\mathrm{D}_{0}$ & $(\mathrm{E}+\mathrm{P})$ & $\mathrm{D}_{1}$ & $(\mathrm{P}+\mathrm{O})$ \\
\hline Consistência, $\%$ & 10 & 10 & 10 & 10 \\
Temperatura, ${ }^{\circ} \mathrm{C}$ & 60 & 70 & 70 & $95 / 105$ \\
Tempo, minutos & 30 & 60 & 180 & 120 \\
Pressão Inicial, atm & - & - & - & 0 e 5 \\
$\mathrm{H}_{2} \mathrm{O}_{2}, \mathrm{~kg} / \mathrm{t}$ & - & 3 & - & 3 e 6 \\
$\mathrm{O}_{2}, \mathrm{~kg} / \mathrm{t}$ & - & - & - & 20 \\
$\mathrm{pH}$ & $2,5-3,0$ & $11,0-11,5$ & $3,8-4,2$ & $10,5-11,0$ \\
$\mathrm{NaOH}, \mathrm{kg} / \mathrm{t}$ & - & 10 & - & 10 \\
$\mathrm{H}_{2} \mathrm{SO}_{4}, \mathrm{~kg} / \mathrm{t}$ & Otimizado & - & Otimizado & - \\
$\mathrm{ClO}_{2}, \mathrm{~kg} / \mathrm{t}$ & 5 & - & 5 & - \\
\hline
\end{tabular}

Em que: $\mathrm{kg} / \mathrm{t}=$ Quilograma de reagente por tonelada de polpa seca; $\mathrm{D}_{0}=$ Estágio inicial de dióxido de cloro; $(\mathrm{E}+\mathrm{P})$ $=$ Estágio de extração alcalina com peróxido de hidrogênio; $\mathrm{D}_{1}=$ Estágio intermediário de dióxido de cloro; $(\mathrm{P}+\mathrm{O})=$ Estágio de peróxido de hidrogênio.

Os estágios de branqueamento $\mathrm{D}_{0},(\mathrm{E}+\mathrm{P})$ e $\mathrm{D}_{1}$ foram efetuados em sacos de polietileno com amostras de $300 \mathrm{~g}$ a.s (absolutamente seca) de polpa. O licor de branqueamento foi adicionado à polpa em temperatura ambiente que, posteriormente, foi aquecida conforme temperatura desejada e transferida para um banho com controle de temperatura, no qual foi mantida pelo tempo descrito na Tabela 1 . Terminada a reação, foram extraídas amostras de licor residual das polpas para análises de $\mathrm{pH}$. A polpa foi lavada com o equivalente a $9 \mathrm{~mL}$ de água por grama de polpa absolutamente seca. Para produção de polpa celulósica foram realizadas quatro repetições para cada estágio da sequência de branqueamento $\mathrm{D}_{0}(\mathrm{E}+\mathrm{P}) \mathrm{D}_{1}$ e, através dos testes estatísticos de análise de variância ANOVA, comprovou-se que, para o parâmetro de alvura, as amostras produzidas eram estatisticamente iguais, possibilitando, assim, a continuidade do estudo do último estágio de branqueamento com peróxido.

Para realização do último estágio $(\mathrm{P}+\mathrm{O})$ (que foi realizado em quatro repetições para cada condição) foi utilizado um digestor rotativo laboratorial com quatro cápsulas individuais de aço inox, com capacidade de 1,5 litros cada e ajuste de pressão e temperatura. Em cada cápsula foram colocadas aproximadamente $70 \mathrm{~g}$ a.s. de polpa, sendo adicionada à polpa uma mistura de água (para ajuste da consistência), $\mathrm{NaOH} \mathrm{e}$ $\mathrm{H}_{2} \mathrm{O}_{2}$. Após atingir temperatura desejada, sob o efeito da rotação constante do digestor, foi aplicada a carga de oxigênio mantendo a pressão desejada. Terminada a reação, a pressão do digestor foi aliviada e a polpa retirada da cápsula foi lavada com $9 \mathrm{~mL}$ de água por grama de polpa absolutamente seca. Os estágios de peroxidação sem pressão também foram realizados no mesmo equipamento, sendo o procedimento apenas diferente quanto a não aplicação da carga de oxigênio.

Todos os estágios de peroxidação com e sem pressurização foram realizados em quatro repetições para realização das análises estatísticas.

\section{Métodos de análise da polpa branqueada}

As análises de número kappa, alvura, viscosidade da polpa, confecção de folhas e reversão de alvura foram efetuadas de acordo com os procedimentos descritos pelas normas TAPPI T236 om-99, TAPPI T525 om-92, TAPPI T230 om-99, TAPPI 205 sp-95 e TAPPI UM 200, respectivamente (TECHNICAL ASSOCIATION OF THE PULP AND PAPER INDUSTRY, 2001). 
Para a análise estatística dos resultados, utilizou-se análise de variância (ANOVA), sendo o nível de significância adotado de 5\%. O software estatístico R, versão 3.1.1 foi utilizado para a realização das análises estatísticas.

\section{RESULTADOS E DISCUSSÃO}

\section{Alvura - efeito da carga de peróxido}

Os valores de alvura para a variação de carga de peróxido de hidrogênio (3 kg/t e $6 \mathrm{~kg} / \mathrm{t})$ estão apresentados na Tabela 2.

TABELA 2: Efeito da carga de peróxido de hidrogênio ( $3 \mathrm{~kg} / \mathrm{t} \mathrm{e} 6 \mathrm{~kg} / \mathrm{t})$ sobre a alvura final da polpa.

TABLE 2: Effect of hydrogen peroxide charge $(3 \mathrm{~kg} / \mathrm{t}$ and $6 \mathrm{~kg} / \mathrm{t})$ on pulp final brightness.

\begin{tabular}{|c|c|c|c|c|c|}
\hline \multicolumn{3}{|c|}{ Temperatura de $95^{\circ} \mathrm{C}$} & \multicolumn{3}{|c|}{ Temperatura de $105^{\circ} \mathrm{C}$} \\
\hline \multirow{2}{*}{$\begin{array}{l}\mathrm{H}_{2} \mathrm{O}_{2} \\
(\mathrm{~kg} / \mathrm{t})\end{array}$} & Com Pressão & Sem Pressão & \multirow{2}{*}{$\begin{array}{l}\mathrm{H}_{2} \mathrm{O}_{2} \\
(\mathrm{~kg} / \mathrm{t})\end{array}$} & Com Pressão & Sem Pressão \\
\hline & \multicolumn{2}{|c|}{ Alvura (\%ISO) } & & \multicolumn{2}{|c|}{ Alvura (\%ISO) } \\
\hline 3 & $90,23(0,17)$ & $89,98(0,29)$ & 3 & $90,70(0,26)$ & $90,28(0,72)$ \\
\hline 6 & $91,05(0,19)$ & $90,65(0,25)$ & 6 & $91,03(0,33)$ & $90,78(0,26)$ \\
\hline
\end{tabular}

Em que: $\mathrm{H}_{2} \mathrm{O}_{2}(\mathrm{~kg} / \mathrm{t})=$ Quilograma de peróxido de hidrogênio por tonelada de polpa seca. Os valores entre parênteses representam o desvio padrão amostral.

Através da análise de variância constatou-se que não houve diferença significativa para variação de carga de peróxido para temperatura a $105^{\circ} \mathrm{C}$ tanto com pressão $\left(\mathrm{F}_{3,4}=0,947\right.$; P-value $\left.>5 \%\right)$ quanto sem pressão $\left(\mathrm{F}_{3,4}=0,899 ; \mathrm{P}\right.$-value $\left.>5 \%\right)$. O aumento da carga de peróxido de hidrogênio de $3 \mathrm{~kg} / \mathrm{t}$ para $6 \mathrm{~kg} / \mathrm{t}$ no estágio pressurizado $(\mathrm{P}+\mathrm{O})$ não propiciou o aumento esperado, uma possível explicação para isso foi que neste trabalho a alvura do estágio realizado anteriormente $\left(\mathrm{D}_{1}\right)$ estava muito elevada (88,5\% ISO). Desta maneira, para atingir uma alvura de $90 \%$ ISO é necessária uma pequena dosagem de peróxido de hidrogênio, porém, sabe-se que quando essa dosagem é aumentada a alvura não possui um incremento linear com o aumento da carga no último estágio de branqueamento, justificando um aumento não tão pronunciado nos valores do parâmetro analisado. Resultados para a temperatura de $95^{\circ} \mathrm{C}$ com pressão $\left(\mathrm{F}_{3,4}=0,0536\right.$; $\mathrm{P}$-value $>5 \%)$ e sem pressão $\left(\mathrm{F}_{3,4}=0,147\right.$; P-value $\left.>5 \%\right)$ seguiram a mesma tendência, assim, apesar do teste de variância não ter sido estatisticamente significativo houve incremento de alvura com o aumento da carga. Resultados análogos foram obtidos por Igerud (1992), Germgärd e Norden (1994) e Pessoti, Colodette e Araújo (1997), em que afirmam que o ganho de alvura aumenta com o aumento da dosagem de $\mathrm{H}_{2} \mathrm{O}_{2}$ para uma mesma temperatura.

\section{Alvura - efeito da temperatura}

Os valores de alvura para a variação temperatura $\left(95^{\circ} \mathrm{C}\right.$ e $\left.105^{\circ} \mathrm{C}\right)$ estão apresentados na Tabela 3. 
TABELA 3: Efeito da temperatura $\left(95^{\circ} \mathrm{C}\right.$ e $\left.105^{\circ} \mathrm{C}\right)$ sobre a alvura final da polpa.

TABLE 3: Effect of temperature $\left(95^{\circ} \mathrm{C}\right.$ and $\left.105^{\circ} \mathrm{C}\right)$ on pulp final brightness.

\begin{tabular}{cccccc}
\hline & \multicolumn{2}{c}{ Carga de Peróxido de $3 \mathrm{~kg} / \mathrm{t}$} & \multicolumn{3}{c}{ Carga de Peróxido de $6 \mathrm{~kg} / \mathrm{t}$} \\
\hline \multirow{2}{*}{ Temp. $\left({ }^{\circ} \mathrm{C}\right)$} & \multicolumn{2}{c}{ Sem Pressão } & Com pressão & \multirow{2}{*}{ Temp. $\left({ }^{\circ} \mathrm{C}\right)$} & \multicolumn{2}{c}{ Sem Pressão } & Com pressão \\
\cline { 2 - 6 } \cline { 5 - 6 } & \multicolumn{2}{c}{ Alvura $(\%$ ISO) } & & \multicolumn{2}{c}{ Alvura $(\%$ ISO) } \\
\hline 95 & $89,85(0,29)$ & $90,23(0,17)$ & 95 & $90,63(0,39)$ & $90,95(0,19)$ \\
\hline 105 & $90,17(0,71)$ & $90,70(0,26)$ & 105 & $90,65(0,25)$ & $91,13(0,30)$ \\
\hline
\end{tabular}

Em que: $\mathrm{kg} / \mathrm{t}=$ Quilograma de peróxido de hidrogênio por tonelada de polpa seca. Os valores entre parênteses representam o desvio padrão amostral.

O teste de análise de variância detectou que houve diferença significativa para o aumento de temperatura de $95^{\circ} \mathrm{C}$ para $105^{\circ} \mathrm{C}$ entre as amostras com pressão de oxigênio de 5 atm e carga de peróxido de 3 $\mathrm{kg} / \mathrm{t}\left(\mathrm{F}_{3,4}=0,0141 ; \mathrm{P}\right.$-value $\left.<5 \%\right)$. Conforme citado por Germgärd e Norden (1994), Ragnar (2003), Siqueira e Silva Filho (2003), Mota et al. (2007) e Mota, Ferreira e Carvalho (2007), os resultados de incremento de temperatura aumentaram significativamente os resultados de alvura da polpa final. A explicação para isso é que o aumento da energia cinética das moléculas químicas proporciona maior ativação dos grupos cromóforos, modificando-os e diminuindo a intensidade de absorção da luz.

As amostras foram estatisticamente iguais para a carga peróxido de hidrogênio de $3 \mathrm{~kg} / \mathrm{t}$ sem pressão de oxigênio $\left(\mathrm{F}_{3,4}=0,108 ; \mathrm{P}\right.$-value $\left.>5 \%\right)$. O mesmo comportamento ocorreu para a carga de $6 \mathrm{~kg} / \mathrm{t}$ com pressão de oxigênio $\left(\mathrm{F}_{3,4}=0,181 ; \mathrm{P}\right.$-value $\left.>5 \%\right)$ e sem pressão de oxigênio $\left(\mathrm{F}_{3,4}=0,15 ; \mathrm{P}\right.$-value $\left.>5 \%\right)$. Tal fato foi verificado para todas as polpas acima citadas devido à alvura do estágio com dióxido de cloro $\left(\mathrm{D}_{1}\right)$ que precede o estágio $(\mathrm{P}+\mathrm{O})$ possuir elevado patamar $(88,5 \%$ ISO).

\section{Alvura - efeito da pressão de oxigênio} Tabela 4.

Os valores de alvura para a variação de pressão de oxigênio ( 0 atm e $5 \mathrm{~atm}$ ) estão apresentados na

TABELA 4: Efeito da pressão de oxigênio ( 0 atm e $5 \mathrm{~atm})$ sobre a alvura final da polpa.

TABLE 4: Effect of oxygen pressure $(0 \mathrm{~atm}$ and $5 \mathrm{~atm})$ on pulp final brightness.

\begin{tabular}{ccccc}
\hline \multirow{2}{*}{ Condições } & \multicolumn{5}{c}{ Estágios de Branqueamento } \\
\cline { 2 - 5 } & $\mathrm{D}_{0}$ & $(\mathrm{E}+\mathrm{P})$ & $\mathrm{D}_{1}$ & $(\mathrm{P}+\mathrm{O})$ \\
\hline Consistência, $\%$ & 10 & 10 & 10 & 10 \\
Temperatura, ${ }^{\circ} \mathrm{C}$ & 60 & 70 & 70 & $95 / 105$ \\
Tempo, minutos & 30 & 60 & 180 & 120 \\
Pressão Inicial, atm & - & - & - & 0 e 5 \\
$\mathrm{H}_{2} \mathrm{O}_{2}, \mathrm{~kg} / \mathrm{t}$ & - & 3 & - & 3 e 6 \\
$\mathrm{O}_{2}, \mathrm{~kg} / \mathrm{t}$ & - & - & - & 20 \\
$\mathrm{pH}$ & $2,5-3,0$ & $11,0-11,5$ & $3,8-4,2$ & $10,5-11,0$ \\
$\mathrm{NaOH}_{2} \mathrm{~kg} / \mathrm{t}$ & - & 10 & - & 10 \\
$\mathrm{H}_{2} \mathrm{SO}_{4}, \mathrm{~kg} / \mathrm{t}$ & Otimizado & - & Otimizado & - \\
$\mathrm{ClO}_{2}, \mathrm{~kg} / \mathrm{t}$ & 5 & - & 5 & - \\
\hline
\end{tabular}

Em que: $\mathrm{kg} / \mathrm{t}=$ Quilograma de peróxido de hidrogênio por tonelada de polpa seca. Os valores entre parênteses representam o desvio padrão amostral. 
O teste de análise de variância detectou que houve diferença significativa ao se aplicar pressão de oxigênio entre as amostras com carga de peróxido de $6 \mathrm{~kg} / \mathrm{t}$ e temperatura de $95^{\circ} \mathrm{C}\left(\mathrm{F}_{3,4}=0,0346\right.$; $\mathrm{P}$-value $<5 \%$ ). Esse resultado era esperado, pois a pressão tem grande efeito no branqueamento com peróxido pressurizado. O aumento da pressão aumenta a reatividade do peróxido com os grupos cromóforos. Este aumento pode ser explicado devido à diminuição da degradação do peróxido que pode ocorrer durante o branqueamento e ainda propicia que o peróxido de hidrogênio atinja grupos cromóforos que estão em áreas mais estericamente impedidas. Altas pressões retardam as reações laterais tornando o peróxido mais eficiente e ainda possibilitam melhor difusão da solução de peróxido nas fibras (COLODETTE, 1994; ROBLES; SOUZA; LEPORINI FILHO, 2006). Costa et al. (2009) comparando a sequência D(E+O+P) $\mathrm{DP}$ e $\mathrm{D}(\mathrm{E}+\mathrm{O}+\mathrm{P}) \mathrm{D}(\mathrm{P}+\mathrm{O})$ encontraram que a inclusão de um estágio pressurizado produz um incremento de alvura. De mesmo modo, Kvaerner Pulping Technologies (1996) obteve melhora de alvura em duas unidades e melhor seletividade aplicando pressão de 5 bar. O mesmo efeito não foi expressivo para a amostra utilizando $3 \mathrm{~kg} / \mathrm{t}$ de peróxido de hidrogênio e temperatura de $95^{\circ} \mathrm{C}$, pois, para baixas cargas aplicadas de peróxido, o efeito não é pronunciado, assim, o teste estatístico não detectou uma diferença significativa $\left(\mathrm{F}_{3,4}\right.$ $=0,213 ;$ P-value $>5 \%$ ).

Para as amostras que utilizaram temperatura de $105^{\circ} \mathrm{C}$, tanto para carga de $3 \mathrm{~kg} / \mathrm{t}$ de peróxido de hidrogênio quanto para a carga de $6 \mathrm{~kg} / \mathrm{t}$ não foi verificado grande efeito no uso da pressão utilizando o gás oxigênio. Os resultados da análise estatística dessas condições foram $\left(\mathrm{F}_{3,4}=0,125\right.$; $\mathrm{P}$-value $\left.>5 \%\right) \mathrm{e}$ $\left(\mathrm{F}_{3,4}=0,168\right.$; P-value $\left.>5 \%\right)$, respectivamente. $\mathrm{O}$ aumento da temperatura em $10^{\circ} \mathrm{C}$ ocultou o efeito da pressurização, não diferenciando significativamente a alvura final da polpa celulósica.

\section{Viscosidade - efeito da carga de peróxido de hidrogênio}

Os valores de viscosidade para a variação de carga de peróxido de hidrogênio (3 kg/t e $6 \mathrm{~kg} / \mathrm{t})$ estão apresentados na Tabela 5 .

TABELA 5: Efeito da carga de peróxido de hidrogênio $(3 \mathrm{~kg} / \mathrm{t}$ e $6 \mathrm{~kg} / \mathrm{t})$ sobre a viscosidade final da polpa.

TABLE 5: Effect of hydrogen peroxide charge $(3 \mathrm{~kg} / \mathrm{t}$ and $6 \mathrm{~kg} / \mathrm{t})$ on pulp final viscosity.

\begin{tabular}{|c|c|c|c|c|c|}
\hline \multicolumn{3}{|c|}{ Temperatura de $105^{\circ} \mathrm{C}$} & \multicolumn{3}{|c|}{ Temperatura de $95^{\circ} \mathrm{C}$} \\
\hline \multirow{2}{*}{$\begin{array}{l}\mathrm{H}_{2} \mathrm{O}_{2} \\
(\mathrm{~kg} / \mathrm{t})\end{array}$} & Com Pressão & Sem Pressão & \multirow{2}{*}{$\begin{array}{l}\mathrm{H}_{2} \mathrm{O}_{2} \\
(\mathrm{~kg} / \mathrm{t})\end{array}$} & Com Pressão & Sem Pressão \\
\hline & \multicolumn{2}{|c|}{ Viscosidade (cP) } & & \multicolumn{2}{|c|}{ Viscosidade (cP) } \\
\hline 3 & $16,65(0,93)$ & $17,05(0,47)$ & 3 & $17,10(1,12)$ & $17,20(0,37)$ \\
\hline 6 & $15,83(0,15)$ & $15,68(0,28)$ & 6 & $16,70(1,53)$ & $16,03(0,86)$ \\
\hline
\end{tabular}

Em que: $\mathrm{kg} / \mathrm{t}=$ Quilograma de peróxido de hidrogênio por tonelada de polpa seca. Os valores entre parênteses representam o desvio padrão amostral.

Através da análise de variância constatou-se que houve diferença significativa na viscosidade na aplicação da carga de peróxido de hidrogênio $(3 \mathrm{~kg} / \mathrm{t}$ e $6 \mathrm{~kg} / \mathrm{t})$ para temperatura de $95^{\circ} \mathrm{C}$ com pressão de oxigênio $\left(\mathrm{F}_{3,4}=0,00276\right.$; P-value $\left.<5 \%\right)$. O incremento da carga de peróxido de hidrogênio aumenta a degradação dos carboidratos, devido ao enriquecimento dos radicais livres formados durante o branqueamento com peróxido de hidrogênio. Resultado similar foi encontrado por Pessoti, Colodette e Araújo (1997) e Eiras et al. (2008), em que a perda de viscosidade aumentou significativamente com o uso de maior dosagem de $\mathrm{H}_{2} \mathrm{O}_{2}$.

No caso das viscosidades das amostras de polpa celulósica que foram branqueadas sem a utilização da pressão de oxigênio não houve diferença estatística significativa tanto para as polpas tratadas com temperatura de $95^{\circ} \mathrm{C}\left(\mathrm{F}_{3,4}=0,487\right.$; P-value $\left.>5 \%\right)$ quanto para as polpas tratadas com temperatura de $105^{\circ} \mathrm{C}\left(\mathrm{F}_{3,4}=0,847\right.$; P-value $\left.>5 \%\right)$. Sem a pressurização, o choque entre as moléculas (radicais livres e carboidratos) são menores levando a uma menor degradação dos carboidratos. 


\section{Viscosidade - efeito da temperatura}

Os valores de viscosidade para as variações de temperatura $\left(95^{\circ} \mathrm{C}\right.$ e $\left.105^{\circ} \mathrm{C}\right)$ estão apresentados na Tabela 6 .

TABELA 6: Efeito da temperatura $\left(95^{\circ} \mathrm{C}\right.$ e $\left.105^{\circ} \mathrm{C}\right)$ sobre a viscosidade final da polpa.

TABLE 6: Effect of temperature $\left(95^{\circ} \mathrm{C}\right.$ and $\left.105^{\circ} \mathrm{C}\right)$ on pulp final viscosity.

\begin{tabular}{|c|c|c|c|c|c|}
\hline \multicolumn{3}{|c|}{ Carga de Peróxido $3 \mathrm{~kg} / \mathrm{t}$} & \multicolumn{3}{|c|}{ Carga de Peróxido $6 \mathrm{~kg} / \mathrm{t}$} \\
\hline \multirow{2}{*}{$\begin{array}{l}\text { Temp. } \\
\left({ }^{\circ} \mathrm{C}\right)\end{array}$} & Sem Pressão & Com Pressão & \multirow{2}{*}{$\begin{array}{c}\text { Temp. } \\
\left({ }^{\circ} \mathrm{C}\right)\end{array}$} & Sem Pressão & Com Pressão \\
\hline & \multicolumn{2}{|c|}{ Viscosidade (cP) } & & \multicolumn{2}{|c|}{ Viscosidade (cP) } \\
\hline 95 & $17,20(0,32)$ & $17,20(1,02)$ & 95 & $15,78(0,87)$ & $16,70(1,32)$ \\
\hline 105 & $17,08(0,44)$ & $16,65(0,81)$ & 105 & $15,68(0,24)$ & $16,08(0,38)$ \\
\hline
\end{tabular}

Em que: $\mathrm{kg} / \mathrm{t}=$ Quilograma de peróxido de hidrogênio por tonelada de polpa seca. Os valores entre parênteses representam o desvio padrão amostral.

No caso das viscosidades das amostras de polpa celulósica, que foram branqueadas com diferentes temperaturas não houve diferença significativa conforme análise estatística. A temperatura aumentou o choque entre as moléculas (radicais livres e carboidratos) de forma a não apresentar diferença na degradação dos carboidratos, medida indiretamente pela viscosidade. Para uma carga de peróxido de hidrogênio de 3 $\mathrm{kg} / \mathrm{t}$, com e sem pressão encontrou-se, pelo teste estatístico, $\left(\mathrm{F}_{3,4}=0,207 ; \mathrm{P}\right.$-value $\left.>5 \%\right)$ e $\left(\mathrm{F}_{3,4}=0,941\right.$; P-value $>5 \%$ ), respectivamente. Para aplicação de uma carga de peróxido de hidrogênio de $6 \mathrm{~kg} / \mathrm{t}$, os testes estatísticos apresentaram-se $\left(\mathrm{F}_{3,4}=0,0655\right.$; P-value $\left.>5 \%\right)$ e $\left(\mathrm{F}_{3,4}=0,0746\right.$; P-value $\left.>5 \%\right)$, sem pressão e com pressão de oxigênio, respectivamente. Resultados diferentes foram encontrados por Pereira (1995) estudando o branqueamento de polpa kraft de eucalipto com peróxido pressurizado cujo aumento da temperatura na faixa de $90-110^{\circ} \mathrm{C}$ produz decréscimo da viscosidade. $\mathrm{O}$ mesmo ocorreu com Pessoti, Colodette e Araújo (1997) e Milanez, Colodette e Carvalho (2008), pois o aumento da temperatura levou a maiores perdas de viscosidade.

\section{Viscosidade - efeito da pressão de oxigênio} na Tabela 7.

Os valores de viscosidade para a variação de pressão de oxigênio ( 0 atm e $5 \mathrm{~atm})$ estão apresentados

TABELA 7: Efeito da pressão de oxigênio ( 0 atm e 5 atm) sobre a viscosidade final da polpa.

TABLE 7: Effect of oxygen pressure ( $0 \mathrm{~atm}$ and $5 \mathrm{~atm})$ on pulp final viscosity.

\begin{tabular}{cccccc}
\hline & \multicolumn{2}{c}{ Carga de Peróxido $3 \mathrm{~kg} / \mathrm{t}$} & & \multicolumn{2}{c}{ Carga de Peróxido $6 \mathrm{~kg} / \mathrm{t}$} \\
\hline \multirow{2}{*}{$\begin{array}{c}\text { Pressão } \\
(\mathrm{atm})\end{array}$} & \multicolumn{2}{c}{$95^{\circ} \mathrm{C}$} & $105^{\circ} \mathrm{C}$ & Pressão & \multicolumn{2}{c}{$95^{\circ} \mathrm{C}$} & $105^{\circ} \mathrm{C}$ \\
\cline { 2 - 3 } \cline { 5 - 7 } & \multicolumn{2}{c}{ Viscosidade $(\mathrm{cP})$} & 0 & \multicolumn{2}{c}{ Viscosidade $(\mathrm{cP})$} \\
\hline 0 & $16,73(0,71)$ & $17,00(0,98)$ & $0,50(0,58)$ & $15,58(0,15)$ \\
\hline 5 & $17,68(0,66)$ & $16,70(0,41)$ & 5 & $17,23(1,02)$ & $15,93(0,10)$ \\
\hline
\end{tabular}

Em que: $\mathrm{kg} / \mathrm{t}=$ Quilograma de peróxido de hidrogênio por tonelada de polpa seca. Os valores entre parênteses representam o desvio padrão amostral.

Através da análise de variância constatou-se que houve diferença significativa de viscosidade pelo efeito da pressurização para temperatura de $95^{\circ} \mathrm{C}$ e carga de peróxido de $6 \mathrm{~kg} / \mathrm{t}\left(\mathrm{F}_{3,4}=0,0346\right.$; P-value < $5 \%$ ). A pressurização do sistema aumenta o choque entre as moléculas levando a uma maior degradação 
dos carboidratos. Resultados semelhantes foram encontrados por Barna et al. (1996) citado por Malmberg e Edwards (2007), Mota et al. (2007) e Mota, Ferreira e Carvalho (2007), os quais verificaram que, quanto maior o tempo de pressurização do sistema, menor a viscosidade encontrada.

No caso da viscosidade da amostra de polpa celulósica branqueada com $3 \mathrm{~kg} / \mathrm{t}$ e temperatura de $95^{\circ} \mathrm{C}$, não houve diferença significativa na análise de viscosidade $\left(\mathrm{F}_{3,4}=0,346\right.$; P-value $\left.>5 \%\right)$. Mesmo com o aumento da temperatura para $105^{\circ} \mathrm{C}$ não foi observada diferença significativa nas viscosidades das polpas tanto para carga de peróxido de hidrogênio de $3 \mathrm{~kg} / \mathrm{t}\left(\mathrm{F}_{3,4}=0,125 ; \mathrm{P}\right.$-value $\left.>5 \%\right)$ quanto para carga de peróxido de hidrogênio de $6 \mathrm{~kg} / \mathrm{t}\left(\mathrm{F}_{3,4}=0,168 ; \mathrm{P}\right.$-value $\left.>5 \%\right)$.

\section{Reversão de alvura}

Neste trabalho, a reversão de alvura não variou significativamente com a temperatura, pressão de oxigênio e carga de peróxido de hidrogênio. Todas as reversões obtidas foram consideradas de baixo percentual, conforme apresentado na Tabela 8.

TABELA 8: Reversão de alvura (\% ISO) para o estágio de peroxidação com e sem pressurização.

TABLE 8: Brightness reversion (\% ISO) to the peroxide stage with and without pressurization.

\begin{tabular}{|c|c|c|c|c|c|c|}
\hline Temp. $\left({ }^{\circ} \mathrm{C}\right)$ & $\mathrm{H}_{2} \mathrm{O}_{2}(\mathrm{~kg} / \mathrm{t})$ & Com pressão & Sem pressão & $\mathrm{H}_{2} \mathrm{O}_{2}(\mathrm{~kg} / \mathrm{t})$ & Com pressão & Sem pressão \\
\hline 95 & \multirow{2}{*}{3} & $1,66(0,04)$ & $1,49(0,03)$ & \multirow{2}{*}{6} & $1,65(0,06)$ & $1,49(0,03)$ \\
\hline 105 & & $1,61(0,02)$ & $1,59(0,02)$ & & $1,60(0,02)$ & $1,65(0,06)$ \\
\hline
\end{tabular}

Em que: $\mathrm{H}_{2} \mathrm{O}_{2}(\mathrm{~kg} / \mathrm{t})$ = Quilograma de peróxido de hidrogênio por tonelada de polpa seca. Os valores entre parênteses representam o desvio padrão amostral.

Os resultados de peróxido pressurizado obtidos por Comelato (2011) foram muito semelhantes aos encontrados neste trabalho, sendo os resultados de reversão de alvura apresentados pela autora menores que uma sequência referência com estágio final de dioxidação. Segundo Colodette et al. (1993), o menor valor de reversão de alvura da polpa tratada com estágio final $(\mathrm{P}+\mathrm{O})$ pode ser explicado pela alta estabilidade de alvura proporcionada pelo peróxido de hidrogênio presente neste estágio.

\section{CONCLUSÕES}

O aumento da carga de peróxido de hidrogênio não teve efeito significativo na alvura das amostras estudadas para estágio com peróxido pressurizado.

$\mathrm{O}$ aumento de $10^{\circ} \mathrm{C}$ na temperatura teve efeito significativo na polpa com tratamento pressurizado a $5 \mathrm{~atm}$ e carga de peróxido de hidrogênio de $3 \mathrm{~kg} / \mathrm{t}$. Para a pressão, o efeito também foi significativo para polpa com tratamento de $95^{\circ} \mathrm{C}$ e carga de peróxido de $6 \mathrm{~kg} / \mathrm{t}$.

Para o parâmetro viscosidade, o aumento da carga de peróxido teve efeito significativo na polpa com pressurização de $5 \mathrm{~atm}$ e temperatura de $95^{\circ} \mathrm{C}$. Para a pressão de oxigênio, o efeito também foi significativo para a polpa com tratamento de $95^{\circ} \mathrm{C}$ e carga de peróxido de hidrogênio de $6 \mathrm{~kg} / \mathrm{t}$.

$\mathrm{O}$ aumento de $10^{\circ} \mathrm{C}$ na temperatura não teve efeito significativo na viscosidade das amostras estudadas para o estágio com peróxido pressurizado.

A reversão de alvura não variou significativamente com a temperatura, pressão de oxigênio e carga de peróxido de hidrogênio. 


\section{REFERÊNCIAS}

COLODETTE, J. L. Peroxide delignification and bleaching. In: ANNUAL NON-CHLORINE BLEACHING CONFERENCE, 3., 1994, Amelia Island. Proceedings... Amelia Island: [s. n.], 1994.

COLODETTE, J. L. et al. Aumentando a seletividade e eficiência no branqueamento com oxigênio pelo uso do metanol. O Papel, São Paulo, v. 54, n. 4, p. 26-36, 1993.

COMELATO, J. S. Efeito de reagentes de branqueamento nas propriedades físicas e mecânicas da polpa de celulose kraft de eucalipto. 2011. 113 f. Dissertação (Mestrado em Engenharia Mecânica Materiais Lignocelulósicos) - Faculdade de Engenharia do Campus de Guaratinguetá, Universidade Estadual Paulista, Guaratinguetá, 2011.

COSTA, M. M. et al. Low environmental impact bleaching sequences for attaining high brightness level with eucalyptus spp pulp. Brazilian Journal of Chemical Engineering, São Paulo, v. 26, n. 1, p. 11-22, Jan./Mar. 2009.

EIRAS, K. M. M. et al. New insights on brightness stability of eucalyptus kraft pulp. Nordic Pulp and Paper Research Journal, Sundsvall, v. 23, n. 1, p. 102-107, Jan. 2008.

GERMGÄRD, U.; NORDEN, S. OZP-Bleaching of kraft pulps to full brightness. In: INTERNATIONAL PULP BLEACHING CONFERENCE, 1994, Vancouver. Proceedings... Montreal: CPPA, 1994.

IGERUD, L. Mill experiences of Lignox bleaching. In: SUNDS DEFIBRATOR TECHNICAL SEMINAR, 9., 1992, Williamsburg. Proceedings... Williamsburg: [s. n.], 1992. p. 1-12.

KVAERNER PULPING TECHNOLOGIES AB (Sweden). Petter Tibbling; Ulla Ekström; Erik Nilsson; Lars-Ove Larsson. Process for peroxide bleaching of chemical pulp in a pressurized bleach vessel. US 5571377A, 22 Dec. 1994, 5 Nov. 1996.

MALMBERG, B.; EDWARDS, L. Dynamic modeling of pressurized peroxide stages with application to full bleach plant simulation. Tappi Journal, Atlanta, v. 6, n. 2, p. 9-17, Apr. 2007.

MILANEZ, A. F.; COLODETTE, J. L.; CARVALHO, A. M. M. L. Condições ótimas para branquear polpa kraft de eucalipto com uma seqüência de três estágios. Scientia Forestalis, Piracicaba, v. 36, n. 78, p. 105113, jun. 2008.

MOTA, S. F. et al. Pressurized hydrogen peroxide bleaching of Eucalyptus globulus pulps. Part I: effect of process variables. Nordic Pulp and Paper Research Journal, Sundsvall, v. 22, n. 1, p. 17-22, Jan. 2007. MOTA, S. M. F.; FERREIRA, L. M. G. A.; CARVALHO, M. G. V. S. Pressurized hydrogen peroxide bleaching of Eucalyptus globulus pulps. Part two: kinects. Nordic Pulp and Paper Research Journal, Sundsvall, v. 22, n. 1, p. 23-27, Jan. 2007.

NAVARRO, R. M. S. Estudo dos diferentes tipos de processos de branqueamento de celulose objetivando a comparação entre seus métodos e a geração do potencial de poluentes em seus respectivos efluentes. 2004. 111 f. Dissertação (Mestrado em Engenharia Química) - Faculdade de Engenharia Química, Universidade Estadual de Campinas, Campinas, 2004.

PEREIRA, E. R. Branqueamento de polpa kraft de eucalipto com perácidos e peróxido pressurizado. 1995. 70 f. Dissertação (Mestrado em Ciência Florestal) - Universidade Federal de Viçosa, Viçosa, MG, 1995.

PESSOTI, J. P.; COLODETTE, J. L.; ARAÚJO, G. A. T. Otimização da sequência O/ODED(PO)D de um processo industrial de branqueamento de polpa kraft de eucalipto. In: CONGRESSO ANUAL DE CELULOSE E PAPEL, 30., 1997, São Paulo. Anais... São Paulo: ABTCP, 1997. p. 149-159.

RAGNAR, M. Alkaline extraction and a control strategy for the chlorine dioxide charge to the final stage in DED bleaching. Nordic Pulp and Paper Research Journal, Sundsvall, v. 18, n. 2, p. 162-167, Feb. 2003. ROBLES, Y. A. M.; SOUZA L. C.; LEPORINI FILHO, C. Avaliação de diferentes tecnologias de branqueamento para obtenção de polpa kraft de eucalipto. O Papel, São Paulo, v. 67, n. 7, p. 62-78, jul. 2006.

SIQUEIRA, J. L. D.; SILVA FILHO, L. L. Branqueamento de polpa Kraft de eucalipto-O papel do peróxido de hidrogênio. In: COLÓQUIO INTERNACIONAL SOBRE CELULOSE KRAFT DE EUCALIPTO, 2003, Viçosa, MG. Anais... Viçosa, MG: UFV, 2003. CD-ROM. 
TECHNICAL ASSOCIATION OF THE PULP AND PAPER INDUSTRY. Testing Procedures of Technical Association of the Pulp and Paper Industry. In: Standard Method. Atlanta: TAPPI, 2001. CDROM.

VENSON, I. Estudos em deslignificação de polpas kraft de Pinus spp. com oxigênio e peróxido. 2008. 136 f. Tese (Doutorado em Engenharia Florestal) - Setor de Ciências Agrárias, Universidade Federal do Paraná, Curitiba, 2008. 